\title{
El rol de las actitudes en la inserción laboral de los desempleados mayores de 45 años*
}

\section{The Role of Attitudes in the Labor Insertion of the Unemployed over 45 Years}

Recibido: octubre 4 de 2011 | Revisado: junio 6 de 2012 | Aceptado: octubre 4 de 2012

\author{
TOMÁs IZQUIERDO RUS ** \\ OLIVIA LÓPEZ MARTÍNEZ *** \\ Universidad de Murcia, Murcia, España
}

doi:10.11144/Javeriana.UPSY12-3.rail

Para citar este artículo: Izquierdo, T. \& López, O. (2013). El rol de las actitudes en la inserción laboral de los desempleados mayores de 45 años. Universitas Psychologica, 12(3), 911-922. doi:10.11144/Javeriana. UPSY12-3.rail

* Artículo de investigación.

** Universidad de Murcia, España. Departamento de Métodos de Investigación y Diagnóstico. Campus Universitario de Espinardo s/n 30100 Murcia (España). Teléfono: +34 868 884553. ResearcherID: I-2198-2013. E-mail: tomasizq@um.es

*** Universidad de Murcia, España. Departamento de Psicología Evolutiva y de la Educación. Campus Universitario de Espinardo s/n 30100 Murcia (España).Teléfono: +34868 884040.E-mail: olivia@ um.es

\begin{abstract}
RES UMEN
La edad se convierte en una de las variables que modula los efectos de la situación de desempleo. Las investigaciones que analizan los efectos del desempleo en los mayores de 45 años muestran que las consecuencias se hacen más notables en este grupo de edad. Se exploraron las actitudes hacia el trabajo de una muestra integrada por 262 personas desempleadas del sur de España. El trabajo concluye con propuestas de intervención y nuevas líneas de investigación en el área.

Palabras clave autores

Actitudes, desempleo, inserción laboral, mayores de 45 años.

Palabras clave descriptores

Psicología Organizacional, investigación cualitativa, actitudes, España.
\end{abstract}

\footnotetext{
A B S T R A C T

Age becomes one of the variables that modulate the effects of unemployment. The studies that analyze the effects of unemployed over 45 years show that the consequences made are more noticeable in this age group. The attitudes toward work were explored using a sample consisted on 262 unemployed people who lives in the south of Spain. The work concludes with proposals for intervention and future research in the area.

Key words authors Attitude, Unemployment, Labor Insertion, People Over 45.

Key words plus

Organizational Psychology, Qualitative Research, Attitude, Spain.
} 


\section{Introducción}

El trabajo ha sido considerado tradicionalmente como el lazo más fuerte de unión entre el individuo y la realidad. La pérdida de ese vínculo de unión, la imposibilidad de jugar un rol para el que se está preparado o su finalización brusca o involuntaria, deja al individuo en una situación más que preocupante. El trabajo, pues, produce ciertos beneficios de los que se ven privados los desempleados (Blanco, 1997).

Al estudiar los diversos modelos teóricos que abordan el fenómeno del desempleo (Izquierdo, 2005), se puede concluir que estos conceden un escaso valor a los factores económicos, políticos y socioculturales y prestan demasiada atención a los aspectos psicológicos, perdiendo de vista la influencia que dichos factores ejercen en los planes de acción de las personas desempleadas.

Es de gran importancia considerar que los efectos psicosociales derivados del desempleo han de estar estrechamente ligados al mundo del trabajo ya que, como apunta Rodríguez (1998), la influencia del trabajo es tal que se manifiesta en el descubrimiento de la identidad de una persona, en el estatus que ocupa en la sociedad, en los niveles remunerativos, en las satisfacciones psicológicas, entre otras.

Para Blanch (2001), el trabajo, además de un dispositivo de carácter económico, se convierte en un mecanismo para el establecimiento de las relaciones sociales interpersonales, un medio de participación cívica, una fuente del bienestar subjetivo, un contexto para la socialización secundaria y un punto de articulación entre la vida pública y la privada, así como entre los proyectos individuales y los colectivos. Se hace pues relevante, tanto a nivel social como científico, comprender cuáles son los significados del trabajo en la sociedad actual, marcada fundamentalmente por la multidiversidad, y cuáles son los sentidos personales engendrados por quienes viven de él (Da Rosa, Chalfin, Baasch \& Scares, 2011).

Diversos estudios (Álvaro, 1992; Álvaro \& Garrido, 2003; Buendía, 1990; García, 1993; M. Garrido, 1995; A. Garrido, 1996; Izquierdo, 2008) abordan la problemática del desempleo y su reper- cusión desde el ámbito de la psicología social, en tanto el debate sobre esta cuestión se ha centrado fundamentalmente en el análisis de los factores económicos que lo provocan y de las políticas económicas necesarias. Esto ha llevado al olvido las importantes consecuencias psicosociales que el desempleo ocasiona en aquellas personas que lo sufren.

Estas consecuencias se hacen más explicitas en los grupos de mayor vulnerabilidad: un alto porcentaje de niños ven mermados sus derechos debido a la necesidad de incorporarse al mercado de trabajo, las mujeres sufren una inserción laboral menos favorable que la población masculina y no menos acuciada es la inserción laboral de las personas de mayor edad.

Autores como García y García (2008) y A. Garrido (2006) consideran que la experiencia psicológica del desempleo varía en función de una serie de variables como la clase social, el género, el nivel de estudios, la edad, el apoyo social o la duración del periodo de desempleo, que determinan una actitud diferenciada hacia el trabajo de las personas en situación de desempleo. Estas variables se acentúan en aquellos grupos con mayores dificultades de inserción laboral.

Izquierdo (2007) analizó las características sociales y psicológicas que repercuten en las probabilidades de encontrar trabajo en uno de estos colectivos con mayor dificultad: los desempleados mayores de 45 años. Señala que determinadas características condicionan la inserción laboral de este grupo de edad: el ámbito laboral, la formación y cualificación, los procesos de aprendizaje, el absentismo por razón de enfermedad y el desempleo de larga duración. De esta forma, la edad se convierte en una de las variables que modula los efectos de la situación de desempleo (Izquierdo, 2010).

Otros autores se han centrado en investigar las consecuencias generadas de la situación de desempleo centrándose fundamentalmente en el análisis de las actitudes o la identidad social de los desempleados. En este sentido, Bauder (2006) señala que las actitudes hacia el empleo difieren entre sociedades y grupos sociales, adquiriendo gran importancia los valores culturales que emanan de la idiosincrasia de cada lugar y, más específicamente, de los atributos de las personas que conforman cada territorio. 
Estudiar el papel de las actitudes en la inserción laboral de las personas desempleadas juega un papel importante por varias razones. Por un lado, porque las actitudes influyen en el comportamiento (Baron \& Byrne, 2002); y, por otro, por la enorme influencia en la inserción o reinserción laboral de los desempleados más desfavorecidos.

La manifestación de determinadas actitudes es fundamental en la inserción profesional, ya que toda persona que emprenda una búsqueda activa de empleo pondrá en marcha toda una serie de conductas con vistas a lograr su inserción laboral. Montilla (2004) afirma que aquellas personas con un alto nivel de disposición interna hacia la acción de búsqueda de empleo pondrán en marcha más conductas y encontrarán trabajo antes que aquellas otras con niveles de disposición medios o bajos.

La conducta de búsqueda de empleo, en muchas ocasiones, escapa del control de las personas, por lo que en este trabajo se consideró el modelo de actitudes según la teoría de la acción planificada (Ajzen \& Madden, 1986) como el más apropiado para entender las actitudes en el ámbito de la inserción profesional. Dicha teoría fue formulada para explicar cómo las metas y los planes guían el comportamiento. Ampliamente estudiada desde la psicología social, plantea que la mayor parte de la conducta humana está fuera del dominio de la persona, quien no puede controlar la aparición o no de la conducta.

Es posible que las personas que sienten que no tienen control sobre la práctica de una conducta de búsqueda de empleo se abstengan de realizarla o no seguirán sus intenciones de ejecutar dicha conducta, no importando sus actitudes y normas subjetivas al respecto. Sin embargo, es más probable que una persona que se siente confiada en su habilidad de realizar la conducta intente realizarla y/o mantenga sus intenciones de hacerlo (Smith \& Stasson, 2000).

Desde que fue descrito este modelo, su aplicación a distintas áreas de la conducta ha sido diversa, siendo el estudio de la búsqueda de empleo uno de los ámbitos de mayor aplicación en los últimos años. Siguiendo el planteamiento de la teoría de la acción planificada, Zikic y Saks (2009) afirman, a partir de una muestra de desempleados, que la intención es el predictor más inmediato de la conducta de búsqueda de empleo.

Desde el ámbito de la orientación profesional, al hablar de la motivación se ha de considerar todo un conjunto de creencias, expectativas y actitudes de los sujetos ante el empleo. Según considera De Pablo (1996), para lograr la inserción laboral no basta con formarse en conocimientos, habilidades y destrezas, sino que es necesario también poseer una actitud que favorezca la motivación y autorresponsabilización de la persona para buscar y encontrar un empleo.

Martínez (2009) afirma que los orientadores intentan, ante esta situación, ajustar las actitudes que de sus usuarios con respecto al empleo, de una situación que ellos juzgan de pasividad (desmoralización, o apatía con el empleo) hacia una de buscador activo, que es más propia del discurso de la empleabilidad en el que se mueven estas medidas.

El propósito del presente trabajo fue analizar la repercusión de las características actitudinales de los desempleados mayores de 45 años en sus probabilidades de encontrar empleo. Además de las condiciones del mercado laboral y las preferencias de los empleadores por determinados grupos de la población, el problema de investigación se podría sintetizar en la siguiente pregunta: ¿Determinan las actitudes la inserción laboral de los desempleados 45 años?

Resolver este interrogante aportaría los elementos fundamentales sobre los que debe basarse la elaboración de acciones dirigidas a personas situadas dentro de los grupos considerados más desfavorecidos ante el desempleo.

\section{Método}

El apartado anterior ha permitido un acercamiento al papel de las actitudes en las personas en situación de desempleo. La investigación empírica pretende un acercamiento a la realidad del fenómeno desde la propia experiencia de las personas. Se ha optado por un diseño de corte cualitativo, concretamente el método fenomenográfico, ya que hace énfasis en descubrir y describir lo particular (León \& Montero, 2010). A continuación se describen las 
características de los participantes, el instrumento de recolección de datos y el procedimiento seguido.

\section{Participantes}

Participaron en la investigación 262 personas inscritas como demandantes de empleo en diversos municipios de la Comunidad Autónoma de Andalucía. Se utilizó la base de datos informatizada de las oficinas de empleo, previa autorización de la dirección del Servicio Público de Empleo Estatal, con el objetivo de seleccionar los demandantes de empleo que formarían parte de la investigación. Los datos más significativos de los participantes se muestran a continuación:

- Existió igual proporción de hombres y mujeres (50\%).

- Respecto al nivel educativo, predominaron las personas con Formación Profesional (I) o equivalente (31.3\%). En menor porcentaje se encontraban aquellas que no saben leer o escribir (12.5 \%), Certificado de Escolaridad (12.5\%), Estudios Primarios, Educación General Básica (25\%), Bachillerato o equivalente (6.3\%), Formación Profesional (II), Ciclo Formativo de Grado Medio (6.3 \%) y Diplomatura (6.3 \%).

- El tiempo de duración del desempleo se repartió de la siguiente forma: menos de tres meses (25\%), de 3 a 12 meses (25\%) y más de dos años (50\%).

- Con respecto a la percepción de prestaciones, predominaron los sujetos que no perciben ninguna prestación o subsidio por desempleo (62.5\%).

\section{Instrumento}

El instrumento de recolección de datos fue la entrevista de actitudes hacia el trabajo con el fin de recabar toda la información relativa al propósito de la investigación. La entrevista de actitudes hacia el trabajo se estructura en seis dimensiones, previa comprobación de las condiciones científicas de fiabilidad y validez en el estudio de Izquierdo (2008). La comprobación de la validez del instrumento se evaluó de dos formas: en primer lugar, se facilitó el instrumento a diferentes profesionales de orientación profesional para su valoración, con el fin de determinar la validez de contenido; en segundo lugar, para determinar la validez de constructo, se procedió a la realización de un análisis factorial de componentes principales, con rotación VARIMAX. La adecuación del análisis factorial se comprobó mediante la medida de Kaiser-Meyer-Olkin y la prueba de esfericidad de Bartlett, encontrando las mismas dimensiones que se definieron al diseñar el instrumento. Por último, la consistencia interna del instrumento, mediante el cálculo del coeficiente alfa de Cronbach (0.886), fue alta y permitió evaluar la homogeneidad de las distintas preguntas del instrumento global.

La entrevista, en su totalidad, consta de 33 preguntas que se organizan en torno a las escalas de actitudes hacia el trabajo sobre las que se ha podido ir conociendo las opiniones, pensamientos, creencias, entre otras. Las cuestiones que componen las escalas de actitudes hacia el trabajo se estructuran en torno a las siguientes dimensiones:

\section{Centralidad del empleo}

La mayor parte de las preguntas de escala miden la importancia que los sujetos conceden al empleo, en tanto que valor instrumental y que valencia psicosocial, en las circunstancias contemporáneas de su vida. En el marco de la socialización de los individuos, la centralidad del empleo supone colocar al empleo como valor central de su vida.

\section{Explicación del desempleo: atribución} interna y atribución externa

Esta escala intenta explorar en qué medida aparecen indicios del papel de tales atribuciones causales sobre las probabilidades de salida de ese estado. Se refiere a la explicación que el demandante de empleo da sobre su falta de trabajo. Tienen menos oportunidades de encontrar trabajo quienes culpan de su situación de desempleo a causas externas (crisis económica, política del Gobierno, innovación tecnológica, etc.) que quienes creen que se debe a 
causas internas (limitada experiencia laboral, falta de formación, poco esfuerzo en buscar trabajo, escasa formación sobre el mercado laboral, etc.).

\section{Autoconcepto personal y profesional}

El autoconcepto personal y profesional presenta una serie de respuestas organizadas de los sujetos concernientes a quién, qué, cómo es y cuánto vale uno mismo, en su ámbito personal y profesional. Sobre esa síntesis de percepciones autorreferenciales basa el sujeto parte de su autoimagen, autoestima, autoevaluación, autopresentación y expectativas de desarrollo individual.

\section{Disponibilidad para el empleo}

En esta escala se evalúa la disponibilidad subjetiva hacia al empleo por parte de individuos particulares. Se entiende como la predisposición a emplearse en otras ocupaciones similares o en categorías inferiores o con dificultades geográficas y de horario. Las limitaciones en cuanto al tipo de trabajo, horario, salario, movilidad geográfica harán más difícil el acceso a un puesto de trabajo.

\section{Estilo de búsqueda de empleo}

Las preguntas que componen esta escala, a diferencia del resto, solicitan al demandante que indique si ha realizado recientemente la actividad que se le especifica. Se pretende medir el grado de actividad con que un demandante de empleo desarrolla su vida cotidiana y su búsqueda de empleo. Entendido así, aquellas personas que posean un estilo de búsqueda activa (visitar empresas, enviar currículo, planificar su tiempo, etc.) tendrán mayores posibilidades de encontrar trabajo que una persona que no le dedique tiempo a la búsqueda de empleo.

\section{Procedimiento}

Se contactó a los participantes a través de los servicios de orientación profesional de la Comunidad Autónoma Andaluza. La realización de la entrevistas se llevó a cabo con la colaboración de investigadores, instruidos para esto, tras solicitar el consentimiento previo de los sujetos. La duración de las entrevistas tuvo una media de 30 minutos y fueron grabadas, previa aceptación de los entrevistados. Los participantes fueron informados de los objetivos de la investigación y se les garantizó el anonimato. Los datos obtenidos con la entrevista de actitudes hacia el trabajo fueron analizados con el programa estadístico de análisis cualitativo AQUAD 6.

\section{Resultados}

El análisis de las entrevistas, a través del programa de análisis de datos cualitativos AQUAD, facilitó el establecimiento de códigos y el posterior análisis en profundidad de los mismos. El tipo de análisis está determinado en función de las escalas de actitudes hacia el trabajo:

\section{a.) Centralidad del empleo}

Los comentarios realizados por los participantes hacen referencia, en general, a la importancia del trabajo como medio económico para cubrir sus necesidades básicas, considerando importante tanto el cobrar un salario como tener un empleo estable. Los entrevistados señalan que mediante el trabajo se pueden hacer proyectos (adquirir una vivienda, formación de los hijos...) y ayudar a los demás debido a la seguridad económica que este brinda. De esta forma es posible conocer el importante papel que el trabajo juega en la vida de las personas.

Hay una cosa evidente, sin trabajo hoy día y siempre... hay que vivir $y$, por tanto, hay que comer y a partir de comer vivir no solo es subsistir sino algo más, sencillamente esa es la razón... en mi caso personal quizás no sé que decirte, porque yo soy un poco aleatorio en esos aspectos pero si tener un trabajo siempre implica una cierta seguridad económica que es fundamental por lo que hemos dicho... (3 meses en desempleo)

Otro aspecto a resaltar es la necesidad que se atribuye a tener un trabajo para sentirse útil y realizado constituyéndose, de esta manera, en un 
medio de satisfacción personal. Parece que cuanto más atractivo se percibe el trabajo, mayores son las expectativas de tener un empleo y la motivación también es mayor.

(...) la verdad que me sentía muy bien, y me sentía, algunas veces imprescindible. ( 9 años en desempleo)

Los desempleados afirman que buscan tener trabajo a fin de obtener ingresos económicos para poder vivir, relacionándolo directamente con la satisfacción de las propias necesidades materiales, lo que influye enormemente en la realización de acciones de búsqueda de empleo. También para un número importante de entrevistados el trabajo permite armonizar la convivencia familiar y personal, como forma de sentirse realizado.

Tener un trabajo me aporta satisfacción interna lo primero, decir bueno soy útil a la sociedad, yo no soy un parásito, hay quien le gusta ser parásito, a mí no... yo cuando estoy sin trabajo me gusta levantarme tarde porque me abotargaba y me daba cuenta que está parado. (14 años en desempleo)

Quizás lo más interesante en centralidad del empleo sean las explicaciones que los participantes ofrecen sobre la importancia de tener un puesto de trabajo. Solamente en el número de razones esgrimidas se observa la relevancia que un puesto de trabajo tiene para la persona. Estas razones permiten conocer el valor que el trabajo juega en la vida de las personas y su repercusión sobre la conducta del desempleado ante la búsqueda de empleo.

\section{b) Atribución del desempleo}

Son diversas las causas por las que los sujetos creen estar desempleados. Una de las razones con mayor número de consenso en los entrevistados es la edad. Se trata de una de las atribuciones causales por las que las personas explican su desempleo referidas a la propia persona (internas)

Llega un momento determinado que ya son los años, ya 59 años no es igual que cuando se tienen $25,30 \ldots$ yo en mi caso lo encuentro en la edad. (6 meses en desempleo)

Pues, porque tenía una enfermedad grave, que tenía ya muchos años, eso es una enfermedad, lo mismo que la juventud es una enfermedad pero esa se cura con los años, la vejez o el llegar a cierta edad es una enfermedad que ya con los años va agravándose cada vez más. (14 años en desempleo)

Otra de las causas, aunque con menor número de consenso que la anterior, se debe a la situación económica del país representada, por lo general, en la figura de sus gobernantes políticos. Esta postura, al contrario que la edad, representa un posicionamiento más "externalista" de los desempleados a la hora de explicar el desempleo.

Estoy sin trabajo porque el trabajo ahora mismo está un poco difícil, y a mí me gustaría que los gobiernos se preocupasen ya por las personas mayores, de que no falte un puesto de trabajo... recriminaría a los gobiernos que dieran subvenciones a cambio de puestos de trabajo. (6 meses en desempleo)

Otros criterios que es necesario destacar se refieren a la experiencia laboral y a la especialización profesional como causantes del desempleo. Las respuestas tienden hacia un equilibrio, salvo en la edad, donde estos factores se consideran imprescindibles para tener un puesto de trabajo. Estas explicaciones más "internistas" determinan, en muchos casos, la labor de búsqueda de empleo de los desempleados.

Hoy también te exigen un nivel de estudios muy elevado que yo no tengo, ordenador y cosas de informática que yo no tengo... entonces eso influye mucho. (13 años en desempleo)

Adquiere relevancia la necesidad de realizar cursos de formación ocupacional, bien para recibir la formación necesaria para desempeñar un puesto de trabajo o como vía de reciclaje profesional, ofreciéndole, en muchos casos, la posibilidad de encontrar un trabajo. La formación ocupacional se configura, 
de esta manera, en una alternativa posible para salir de la situación de desempleo.

Pienso que contra más se aporte es mejor para la persona y para todo el mundo. (8 años en desempleo)

Puedes necesitar un curso, aparte del trabajo que tú ya tengas realizado... experimentar otros trabajos distintos nunca viene mal... porque ves que el trabajo está estancado, que no tiene salida, probar otros trabajos a través de estos cursos. (2 meses en desempleo)

Como se observa en las diversas respuestas existe cierta variabilidad en la atribución del desempleo aunque, de forma general, existe cierta tendencia hacia causas de tipo interno. Es más, aquellas atribuciones externas, en ocasiones, tienden a convertirse en individualistas, sobre todo en aquellos desempleados con un mayor tiempo en desempleo.

La atribuciones de tipo externo no hacen más que ocultar el verdadero problema que puede ser de índole personal (ausencia de formación, carencia de habilidades sociales, búsqueda de empleo no bien dirigida...).

\section{c) Autoconcepto personal y profesional}

Básicamente, los sujetos entrevistados han señalado como cualidad principal la capacidad de ejercer algún tipo de trabajo relacionado con su profesión al ofrecer, en muchos casos, experiencia. Se ha observado que la valoración de la experiencia como característica profesional es mayor cuando aumenta la edad. Igualmente, se ha destacado un amplio número de cualidades "implícitas" en las tareas propias de los puestos de trabajo.

Mi experiencia social y también mi experiencia de años de vida... creo que es importante... sobre todo relaciones públicas, me sé desenvolver muy bien y puedo aportar el trato con las personas, buen clima, sería mi especialidad. (14 años en desempleo)

Mi experiencia, en mi trabajo como electricista... lo demostrado, yo he estado aparte dentro de la electricidad en montajes eléctricos, líneas de alta y baja, instalación de pisos, de casas... (7 meses en desempleo)

Desde este planteamiento, cuestiones como la experiencia laboral y la especialización profesional se consideran puntos favorables a la hora de ser contratado. Al igual que la experiencia, ambas cuestiones forman parte de las características profesionales de los demandantes. Nuevamente adquieren importancia estos dos factores al tener un doble protagonismo: en atribución causal y en autoconcepto profesional.

Siempre si se es especialista en algo eso está muy bien mirado... es un hábito normal, creo yo. (8 años en desempleo)

La suerte y las oportunidades fueron también categorías analizadas, aunque en menor grado que las anteriores, quedando, de esta forma, en un segundo plano de importancia. De igual manera, juega un papel importante la inteligencia, el nivel cultural y el carácter personal, aunque quedarían relegadas a los últimos lugares de una jerarquía en autoconcepto personal y profesional. Destaca el 'enchufe' como decisivo, en muchos casos, para encontrar trabajo.

El enchufe si hace mucho, porque eso funciona toda la vida y ahora en los tiempos que corren mucho más que antes. (13 años en desempleo)

Al preguntarles sobre su autoconcepto personal y profesional hemos obtenido unas respuestas que presentan cierta tendencia hacia el plano profesional, si bien existe una gran variedad en las respuestas obtenidas. Curiosamente, estas personas, algunas de las cuales han tenido empleo con anterioridad, valoran en menor medida su autoconcepto personal a la hora de encontrar trabajo.

\section{d) Disponibilidad para el empleo}

Los entrevistados han señalado que les gustaría ejercer una ocupación acorde a su preparación e 
interés profesional, siendo mejor aquella que han desempeñado con anterioridad. Están dispuestos a adaptarse a la mayoría de los requerimientos del puesto de trabajo, siempre y cuando se trate de un trabajo digno y que esté remunerado conforme a la legislación vigente.

Siempre se tiene que tener un poco en cuenta algunos aspectos, pero sobre todo que te encuentres a gusto con el trabajo, con los compañeros y demás... porque en principio todo te puede parecer desconocido pero luego le encuentras siempre aspectos positivos. (6 meses en desempleo)

Hay un equilibrio entre muchas cosas... estar a gusto con el trabajo, lo económico es fundamental, primordial para empezar, no en el sentido economicista del término sino que evidentemente necesitas vivir, pero dentro de eso yo valoro más el estar a gusto. (3 meses en desempleo)

En algunos casos, en mayor número las mujeres, señalan la importancia de tener un trabajo de tiempo parcial o de "pocas horas", a fin de compaginarlo con las tareas domésticas, cuidado de hijos o la atención a personas mayores. Esta relativa disponibilidad se asocia con la centralidad atribuida al trabajo y, en consecuencia, a aspectos como el grado de necesidad económica personal o familiar derivado de la propia situación de desempleo.

Si fuese de media jornada mucho mejor, para que te voy a decir que no... la media jornada me sería ideal porque con la otra media jornada puede trabajar otra persona como yo, ya con media jornada y lo poco de mi marido ya salimos adelante. ( 9 años en desempleo)

Esta actitud se relaciona con los valores laborales, es decir, con la propia concepción de los aspectos más deseables del trabajo y puede dificultar la actividad de búsqueda de empleo. Esta disponibilidad varía según el género, nivel de estudios, etc.

Es necesario ser consciente de que la disponibilidad parcial limita la consecución de un puesto de trabajo. Sirva de ejemplo el caso de personas desempleadas con cargas familiares que se resisten a la idea de tener un trabajo a tiempo completo. A pesar de esta relativa disponibilidad la tendencia en las respuestas de los entrevistados, en general, es clara y se refleja en la alta disposición de los mismos para trabajar.

\section{e) Percepción sobre la contratación de los empresarios}

La categoría conceptual más utilizada para describir el criterio de selección seguido por los empresarios en la selección de los trabajadores es la edad. Los entrevistados piensan que el empresariado, en general, valora más la juventud por lo que ven en la edad el principal sesgo con el que tienen que enfrentarse a la hora de buscar trabajo. Igualmente, consideran que los empresarios valoran el género, la experiencia laboral o las cualidades profesionales, aunque en menor grado que la edad.

Se fijan en la edad porque lo que quieren es meter críos, se aprovechan de ellos y le dan dos perras gordas y con eso están apañaos... y una persona como yo no se conforma con eso, quiere ganar lo suyo. (3 meses en desempleo)

A las mujeres se le pone más la zancadilla que al hombre (...) se fijan más en el hombre que en la mujer, las mujeres estamos más discriminadas hacia el trabajo. (7 años en desempleo)

Aspectos como el tiempo en desempleo, la percepción de prestaciones o la situación económica apenas son considerados por los empresarios, ya que por encima de estas cuestiones buscan su propio beneficio o el de la empresa. Por ello, estos aspectos no se convierten en cuestiones primordiales para discriminar candidatos en las ofertas presentadas por los empresarios.

Los empresarios normalmente en eso no se fijan (...) al empresario lo único que le interesa es encontrar una persona que sustituya el puesto de trabajo que le está haciendo falta, pero ellos no paran a fijarse si llevan más tiempo trabajando o menos tiempo trabajando. (6 meses en desempleo) 
Estas respuestas ayudan a completar la percepción que los desempleados tienen acerca de la contratación de los empresarios; en general, consideran el papel que pueden jugar determinadas características con vistas a su posterior incorporación o reincorporación laboral. Esta visión condiciona, en muchas ocasiones, la conducta llevada a cabo por los demandantes para conseguir trabajo.

\section{f) Estilo de búsqueda de empleo}

Esta escala puede ser analizada en dos sentidos, de acuerdo a las respuestas obtenidas. Por un lado, se encuentran los entrevistados que actualmente no realizan ninguna actividad de búsqueda de empleo y los que, opuestamente, sí lo hacen. Estas posturas ponen de relieve dos situaciones: por un lado, la inexistencia de puestos de trabajo ocupables y, por otro lado, un cierto estilo de búsqueda de empleo.

Últimamente no, ni hacer cursos ni nada por la obligación que tengo (...) cuando voy a las revisiones me paro a ver los cursos y eso pero como no veo posibilidad de compaginarlo con el tiempo que tengo no me dedico mucho. (12 años en desempleo)

Todos los días veo el programa de la 2, que ofrecen muchos trabajos y cursos, cuando abren las bolsas, las mismas compañeras del trabajo, voy al paro y pregunto a ver lo que hay, voy a los sindicatos a ver los cursos (...) porque echo bolsas, hago oposiciones, porque voy al paro, hago todo lo que hace falta. (4 meses en desempleo)

Se puede observar que existe una desmotivación en relación con las actividades para buscar trabajo, los motivos han sido señalados anteriormente como "causas externas", indicando que no hay fuentes de trabajo posibles, que se traduce en la no búsqueda de empleo (al menos en los últimos días). A esto se añaden, en gran parte de los casos, determinadas condiciones referidas a las características del puesto de trabajo, que disminuye considerablemente la búsqueda.

He hecho toda una serie de cosas para ver si realmente encuentro trabajo (...) pero me limito a cosas que ya tengo más o menos seguras (...) se mueve uno dentro de lo realmente factible, de las posibilidades reales que vas a tener, no vas a calentarte la cabeza con cosas que luego no van a salir. (4 meses en desempleo)

Sin embargo, también puede notarse que existe una proporción importante de entrevistados que recurre a todos los medios posibles para conseguir trabajo, notándose acciones como la visita a empresas, el contacto con personas del propio ramo, búsqueda en prensa o la información obtenida en oficinas de empleo como las más utilizadas. Tal vez un mayor éxito en la búsqueda sea el motivo que les lleve a utilizar estas acciones y no otras.

Sí, en la revista Siempre a Mano siempre he estado mirando, incluso en Internet me he metido alguna vez también a ver si hay algo (...) en las veces que he tenido que ir a que me pongan el sello me he fijado en los carteles que había allí puestos a ver si había algo (...) donde he estado yendo ha sido al Ayuntamiento, tienes que personarte donde sea y decir: bueno que, ihay algo? (7 meses en desempleo)

Se podría terminar comprobando que existen dos tendencias en el estilo de búsqueda: una negativa hacia la búsqueda de empleo, relacionada con aspectos de tipo externo; y otra positiva, empleando todos los recursos disponibles que se tiene al alcance. Posicionarse en una tendencia u otra así como la intensidad de utilizar una determinada acción depende de la disposición de cada persona para aceptar un empleo.

\section{Discusión}

Una revisión de los estudios sobre las actitudes en personas desempleadas ha permitido extraer unas primeras conclusiones teóricas relevantes. Diversos autores (Buendía, 2010; García \& García, 2008; A. Garrido, 2006; Izquierdo, 2010; Schafgans, 2000) ponen de manifiesto la existencia de diversos condicionantes en la aparición de actitudes negativas hacia el trabajo.

Los resultados de este estudio evidencian la existencia de una serie de actitudes que condicionan 
la inserción laboral. La situación socioeconómica, los prejuicios, la explotación laboral y la discriminación laboral conforman, según Izquierdo (2008), una actitud en los desempleados que determina la toma de decisiones y, en consecuencia, su inserción o reinserción laboral.

Estudios como los de Izquierdo $(2005,2008)$ e Izquierdo y Alonso (2010) han intentado analizar las actitudes hacia el trabajo en desempleados mayores de 45 años. Sin embargo, de la revisión de la literatura, se constata que las investigaciones y publicaciones sobre el desempleo en este grupo de edad son cuantitativamente menores que las que se orientan hacia otras etapas del ciclo vital (Izquierdo \& Hernández, 2011; Izquierdo, Hernández \& Maquilón, 2010).

El propósito de esta investigación ha sido analizar la incidencia de características actitudinales en la probabilidad de encontrar trabajo en personas desempleadas mayores de 45 años. Con este fin se utilizó la entrevista de actitudes hacia el trabajo, compuesta por seis escalas para evaluar las siguientes dimensiones: centralidad del empleo, atribución causal desempleo, disponibilidad para el empleo, contratación empresarial y estilo de búsqueda de empleo.

Las respuestas obtenidas confirman, en términos generales, una alta centralidad personal del trabajo y una fuerte preocupación por la situación de desempleo así como una valoración muy positiva de su imagen personal. Los entrevistados comparten una nítida tendencia a la atribución interna de las causas del desempleo, y la búsqueda de empleo no es muy amplia. La disponibilidad se caracteriza por el establecimiento de una serie de condiciones y, por último, manifiestan como esenciales una serie de características exitosas en los procesos de selección. Estos datos confirman los resultados obtenidos en otras investigaciones (Izquierdo, 2008, 2010; Martínez, García \& Maya, 2001) que consideran que las probabilidades de encontrar empleo son mayores en aquellas personas que tienen más predisposición para trabajar, siendo superior a la influencia que puedan ejercer otras variables.

De las entrevistas realizadas se puede afirmar que la duración del desempleo afecta de forma ge- neralizada a los desempleados, y que esta influencia es distinta de la que pueden ejercer otras variables como la edad, el género, el nivel de estudios y la percepción de prestaciones. Los datos obtenidos son coincidentes con las investigaciones realizadas por García y García (2008), Izquierdo y Alonso (2010) y Schafgans (2000).

Los resultados de la presente investigación sugieren diversas propuestas, a modo de orientaciones, para mejorar la calidad de la intervención psicosocial y eliminar, de esta forma, las dificultades encontradas en relación con el trabajo de las actitudes con los desempleados de mayor vulnerabilidad. Las más destacadas, según Izquierdo (2010), son las siguientes:

- Una reformulación de los objetivos de la intervención, insistiendo en la necesidad de ayudar a los desempleados a cambiar sus actitudes, especialmente las referidas a la disponibilidad para el empleo y la percepción sobre la contratación de los empresarios.

- Sería necesario garantizar una mayor flexibilidad metodológica con el fin de adecuar los objetivos y las actividades a las circunstancias y características de cada demandante de empleo.

- Esta flexibilidad exige, a su vez, la incorporación de un mayor número de actividades con la intención de seleccionar aquellas que se consideren más adecuadas para la consecución de los objetivos propuestos.

- Por último, se debería ofrecer como modalidad de actuación la individual, por las peculiaridades de los diversos grupos, con apoyo de la modalidad grupal generalmente bien aceptada por los desempleados.

Esta investigación comparte los argumentos de otros estudios científicos (Buendía, 2010) en lo que tiene que ver con la necesidad de tener presente el mercado laboral y describir a los desempleados como aquellos que no han tenido éxito al recurrir a un empleador. De ahí la importancia de considerar a los grupos más desfavorecidos como los principales destinatarios de las políticas activas de empleo. 
Como limitaciones del estudio, cabe destacar que el carácter transversal de la investigación no ha permitido clarificar con profundidad las relaciones existentes entre desempleo y actitudes hacia el trabajo en la población estudiada. Investigaciones posteriores deben orientarse hacia la elección de estudios longitudinales que permitan extraer los mecanismos que hacen del desempleo una experiencia distinta en cada una de las personas afectadas.

Nuevas líneas de investigación sobre las actitudes hacia el trabajo podrían plantear la incorporación de otros grupos sociales y variables que pueden tener un efecto moderador en la inserción laboral, tales como el género, el nivel educativo, los recursos económicos y el apoyo social, que pueden tener un efecto mediador en las personas desempleadas y dar un mayor sentido a la experiencia individual del desempleo. Además, la metodología empleada en esta investigación puede ser útil para el análisis de las actitudes hacia el trabajo derivadas de la situación del desempleo en otros contextos y colectivos, específicamente los más desfavorecidos ante el empleo.

\section{Referencias}

Ajzen, I. \& Madden, T. J. (1986). Prediction of goaldirected behavior: Attitudes, intentions, and perceived behavioral control. Journal of Experimental Social Psychology, 22(5), 453-474.

Álvaro, J. L. (1992). Desempleo y bienestar psicológico. Madrid: Siglo XXI.

Álvaro, J. L. \& Garrido, A. (2003,). Desempleo, salud y exclusión social. Madrid: Siglo XXI.

Baron, R. \& Byrne, D. (2002). Psicología social. Madrid: Prentice-Hall.

Bauder, H. (2006). Origin, employment status and attitudes towards work: Immigrants in Vancouver, Canada. Work, Employement and Society, 20(4), 709-729.

Blanco, A. (1997). Factores psicosociales de la vida adulta. En M. Carretero, J. Palacios \& A. Marchesi (Comps.), Psicología evolutiva. Adolescencia, madurez y senectud (pp. 201-233). Madrid: Alianza.

Blanch, J. M. (2001). Empleo y desempleo: iviejos conceptos en nuevos contextos? En E. Agulló \& A.
Ovejero (Coords.), Trabajo, individuo y sociedad (pp. 27-48). Madrid: Pirámide.

Buendía, J. (1990). Psicopatología del desempleo. Anales de Psicología, 6(1), 21-36.

Buendía, J. (2010). El impacto psicológico del desempleo. Murcia: Editum.

Da Rosa, S., Chalfin, M., Baasch, D. \& Scares, J. (2011). Sentidos y significados del trabajo: un análisis con base en diferentes perspectivas teórico-epistemológicas en Psicología. Universitas Psychologica, 10(1), 175-188.

De Pablo, J. M. (1996). Desarrollo de los aspectos personales para la ocupación: una metodología para el cambio con grupos de desempleados. Intervención Psicosocial, 5(15), 75-101.

García Rodríguez, Y. (1993). Desempleo: alteraciones psicológicas. Valencia: Promolibro.

García, A. M. \& García, M. G. (2008). La influencia de los rasgos psicológicos en las actitudes hacia el empleo. Revista de Psicología del Trabajo y de las Organizaciones, 24(2), 203-233.

Garrido, A. (1996). Psicología social del desempleo. En J. L. Álvaro, A. Garrido \& J. L. Torregrosa (Coords.), Psicología social aplicada (pp. 85-120). Madrid: McGraw-Hill.

Garrido, A. (2006). Sociopsicología del trabajo. Barcelona: Universitat Oberta de Catalunya.

Garrido, M. (1995). Desempleo y psicopatología. Un estudio empírico e interpretación psicodinámica. Valencia: Promolibro.

Izquierdo, T. (2005). Actitudes hacia el trabajo de los desempleados mayores de 45 años. Granada: Editorial Universidad de Granada.

Izquierdo, T. (2007, noviembre). Cultura del desempleo en América del Sur. El caso concreto de los desocupados mayores de 45 años del departamento de Caaguazú (Paraguay). Actas del II Encuentro Internacional de Educación en Valores, Camagüey, Cuba.

Izquierdo, T. (2008). El desempleo en los mayores de 45 años. Jaén: Consejo Económico y Social.

Izquierdo, T. (2010). Los nuevos retos del mercado laboral: una perspectiva desde la orientación profesional. Úbeda, España: Editorial Amarantos.

Izquierdo, T. \& Alonso, H. J. (2010). Valores culturales y consecuencias psicosociales del desempleo en 
América Latina. Revista de Psicología del Trabajo y de las Organizaciones, 26(2), 123-133.

Izquierdo, T. \& Hernández, F. (2011). Orientación profesional y desempleo adulto. En J. Maquilón et al. (Coords.), Cambios educativos y formativos para el desarrollo humano y sostenible (pp. 519-526). Murcia: Editum.

Izquierdo, T., Hernández, F. \& Maquilón, J. J. (2010, septiembre). Psicología del ciclo vital en el desempleo adulto. Actas de I Congreso Internacional de Psicología del Trabajo y de las Organizaciones, Buenos Aires, Argentina.

León, O. \& Montero, I. (2010). Métodos de investigación en psicología y educación. Madrid: McGraw-Hill.

Martínez, A. (2009). Los orientadores laborales. Trabajo cotidiano y efectos sobre sus públicos. Cuadernos de Relaciones Laborales, 27(2), 145-169.

Martínez, M. F., García, M. \& Maya, I. (2001). El rol del apoyo social y las actitudes hacia el empleo en el emplazamiento laboral de inmigrantes. Anuario de Psicología, 32(3), 51-65.

Montilla, S. (2004). Una aproximación a la motivación en procesos de orientación laboral. Revista de Empleo de la Junta de Andalucía, 3(5), 43-44.

Rodríguez, M. L. (1998). La orientación profesional: Vol. 1. Teoría. Barcelona: Ariel.

Schafgans, M. M. A. (2000). Gender wage differences in Malaysia: Parametric and semiparametric estimation. Journal of Development Economics, 63 (2), 351-378.

Smith, B. \& Stasson, M. (2000). A comparison of health behavior constructs: Social psychologycal predictors of AIDS - preventive behavioral intentions. Journal of Applied Social Psychology, 30(3), 443-462.

Zikic, J. \& Saks, A. M. (2009). Job search and social cognitive theory: The role of career-relevant activities. Journal of Vocational Behavior, 74(1), 117-127. 